\title{
Sex-Stratified Trends in Meeting Physical Activity Guidelines, Participating in Sports, and Attending Physical Education Among US Adolescents, Youth Risk Behavior Survey 2009-2019
}

\author{
Tiffany J. Chen, Kathleen B. Watson, Shannon L. Michael, and Susan A. Carlson
}

\begin{abstract}
Background: During the past decade, guidelines for youth aerobic and muscle-strengthening physical activity remained unchanged. Active People, Healthy Nation ${ }^{\text {SM }}$ highlighted school and youth strategies (eg, sports and physical education [PE]) to increase physical activity. Sex, grade, and race/ethnicity disparities exist. This study examines sex-specific trends and differences by grade and race/ethnicity for the prevalence of 5 youth physical activity behaviors from 2009 to 2019. Methods: The national Youth Risk Behavior Survey assesses adolescents (grades 9-12) meeting the aerobic, muscle-strengthening, and both guidelines (2011-2019) and sports participation and daily PE (2009-2019). Sex-stratified logistic regression assessed trends and 2009 or 2011-2019 differences by grade and racial/ethnic subgroups. Results: Decreases in meeting the aerobic, muscle-strengthening, and both guidelines were observed for nearly all male subgroups by grade and race/ethnicity, whereas female subgroups exhibited declines or no change to low prevalence. Sports and PE participation remained mostly constant; select subgroups showed decreases (ie, Hispanic males [sports]; Black males and ninth-grade females [PE]). Conclusions: Past decade prevalence and patterns suggest that school-based and other strategies for all adolescents and tailored interventions for sex-specific subgroups may be needed to supplement sports and PE in promoting high school youth physical activity.
\end{abstract}

Keywords: exercise, health disparities, public health, surveillance

More than a decade ago, the 2008 Physical Activity Guidelines for Americans established the first youth physical activity guidelines. ${ }^{1}$ These guidelines recommended the frequency, duration, intensity, and types of physical activity that children and adolescents need for health benefits, such as improved cardiorespiratory fitness, stronger muscles and bones, healthier levels of body fat and weight, reduced symptoms of anxiety and depression, and fewer chronic disease risk factors, for a better chance at a healthy adulthood. ${ }^{1}$ Although the Physical Activity Guidelines for Americans, second edition, updated the guidance for health-enhancing physical activity in 2018, the youth guidelines did not change; children and adolescents ages 6-17 years should do 60 minutes $(1 \mathrm{~h})$ or more of moderate to vigorous physical activity (MVPA) daily. ${ }^{2}$ In addition, the youth guideline recommends that most of their daily 60 minutes or more is aerobic physical activity, and that children and adolescents include each of the following components on at least 3 days per week: vigorous-intensity aerobic physical activity, muscle-strengthening physical activity, and bonestrengthening physical activity. ${ }^{2}$

Halfway between the first and second editions of the Physical Activity Guidelines for Americans: Strategies to Increase Physical Activity Among Youth identified setting-specific evidence-based interventions to increase youth physical activity. In particular, the

\footnotetext{
Chen, Watson, and Carlson are with the Division of Nutrition, Physical Activity, and Obesity, National Center for Chronic Disease Prevention and Health Promotion, Centers for Disease Control and Prevention, Atlanta, GA, USA. Michael is with the Division of Diabetes Translation, National Center for Chronic Disease Prevention and Health Promotion, Centers for Disease Control and Prevention, Atlanta, GA, USA. Carlson is also with the Division of Population Health, National Center for Chronic Disease Prevention and Health Promotion, Centers for Disease Control and Prevention, Atlanta, GA, USA. Chen (pgi8@cdc.gov) is corresponding author.
}

school setting was highlighted as providing an opportunity to affect a large percentage of youth through multicomponent, schoolbased, physical activity programs, such as activity before and/or after school (eg, sports clubs) and physical education (PE). ${ }^{3}$ The release of the National Youth Sports Strategy in 2019 then highlighted the contribution of sports in fulfilling 60 minutes of daily physical activity for youth, while providing other psychosocial and academic benefits. ${ }^{4}$ Moreover, the Centers for Disease Control and Prevention's Active People, Healthy Nation ${ }^{\text {SM }}$ initiative recognized the importance of both youth sports and PE in its School and Youth Programs strategy for increasing physical activity. ${ }^{5}$ In addition to objectives related to meeting the physical activity guidelines, Healthy People 2030 included national objectives for sports participation and daily PE as strategies to increase youth physical activity. ${ }^{6}$

These guidelines and strategies work to improve youth physical activity within the context of distinct sex disparities, age and grade patterns, and racial/ethnic differences. Research from various data sources has established that more male youth tend to be physically active than female youth ${ }^{7-10}$; both gender norms ${ }^{11}$ and biological age or maturation by $\operatorname{sex}^{12}$ contribute to this disparity. Younger youth also tend to be more physically active than older youth, by age ${ }^{7,10,12,13}$ and by grade. ${ }^{14-20}$ Finally, though literature on youth physical activity by race/ethnicity varies, significant differences have been found, with some racial/ethnic groups getting more physical activity than others. ${ }^{7,8,21}$ Furthermore, neighborhoods considered supportive of physical activity by parents (ie, by perceived safety, sidewalks or walking paths, and parks, playgrounds, or recreation centers) have smaller proportions of non-Hispanic Black and Hispanic adolescents, in which possible crime or how well-maintained neighborhood features are could play a role in parent perceptions of their neighborhoods. ${ }^{22}$ 
Biennially, surveillance summaries on youth risk behaviors report national prevalence estimates and trends for adolescent physical activity, often including sex-stratified estimates by grade and race/ethnicity. ${ }^{9,15-19}$ However, sex-stratified national trends in adolescent physical activity by grade and race/ethnicity have not yet been reported. We examine sex-specific trends and differences from 2009 to 2019 by grade and race/ethnicity in the prevalence of the following youth physical activity behaviors: (1) meeting the aerobic physical activity guideline, (2) meeting the musclestrengthening guideline, (3) meeting both aerobic and musclestrengthening guidelines, (4) participating in sports, and (5) attending daily PE classes. Sex-specific surveillance of youth physical activity is important in identifying subgroups of youth who may benefit from tailored public health and education interventions for promoting physical activity.

\section{Methods}

\section{Study Sample}

The national Youth Risk Behavior Survey (YRBS) is a crosssectional, nationally representative, school-based survey of selfreporting US public and private school students in grades 9 to 12 throughout the 50 states and the District of Columbia. The YRBS is self-administered, conducted biennially, and uses a 3-stage cluster sample design. The first-stage sampling frame of primary sampling units (ie, counties; parts of large counties; or groups of small, adjacent counties), categorized into 16 strata by metropolitan statistical area status and percentages of Black and Hispanic students, is created by sampling primary sampling units using probabilities proportional to their overall school enrollment size. The second-stage sampling frame of secondary sampling units (ie, a school with grades 9-12 or created from combining nearby schools to get all 4 grades) is created by sampling from those primary sampling units, using probabilities proportional to school enrollment size. The third-stage sampling frame is created by randomly sampling 1 or 2 classrooms of a required subject or period in each grade, 9 to 12. All students in each sampled class are eligible for participation; schools, classes, and students that refuse participation are not replaced.

Data from biennial YRBS 2009-2019 (ie, 2009, 2011, 2013, 2015, 2017, and 2019) were obtained to examine trends over time for each of the following measures. For every year of data analyzed, students with missing sex, grade, or race/ethnicity were excluded. Then, for every year of each physical activity behavior analyzed, students with missing responses to the respective physical activity behavior were excluded. Behaviors based on guidelines had data from 2011 to 2019: for youth meeting the aerobic guideline $(n=68,970$, missing $=1858)$, muscle-strengthening guideline $(n=53,084$, missing $=17,744)$, and both aerobic and muscle-strengthening guidelines $(n=65,601$, missing $=5227)$. For the measure related to meeting both aerobic and muscle-strengthening guidelines, respondents who reported not meeting one of the guidelines were counted as not meeting both guidelines, even if they were missing information on the remaining guideline. Behaviors based on strategies had data from 2009 to 2019: for youth participating in sports $(\mathrm{n}=76,263$, missing $=10,606)$ and attending daily PE classes $(n=82,010$, missing $=4859)$. Because of the extra year of data (2009) for sports participation and daily PE attendance, the total number of respondents across all available years (excluding missing demographic data) is different for the guidelines$(70,828)$ and strategies-based behaviors $(86,869)$.

\section{Measures}

Youth Meeting the Aerobic Guideline. To assess students meeting the aerobic guideline, students were asked, "During the past 7 days, on how many days were you physically active for a total of at least 60 minutes per day? (Add up all the time you spent in any kind of physical activity that increased your heart rate and made you breathe hard some of the time.)" The 8 response options were 0 to 7 days. Students were classified as meeting the aerobic guideline ${ }^{2}$ if they selected "7 days."

Youth Meeting the Muscle-Strengthening Guideline. To assess students meeting the muscle-strengthening guideline, students were asked, "During the past 7 days, on how many days did you do exercises to strengthen or tone your muscles, such as pushups, sit-ups, or weightlifting?" The 8 response options were 0 to 7 days. Students were classified as meeting the muscle-strengthening guideline $^{2}$ if they selected a response option of 3 or more days.

Youth Meeting Both Aerobic and Muscle-Strengthening Guidelines. To assess students meeting both aerobic and musclestrengthening guidelines, the responses to the 2 questions above were used. Students were classified as meeting both the aerobic and muscle-strengthening guidelines ${ }^{2}$ if they selected "7 days" to being physically active for a total of at least 60 minutes per day and 3 or more days to exercising to strengthen or tone muscles.

Youth Participating in Sports. To assess students participating in sports, students were asked, "During the past 12 months, on how many sports teams did you play? (Count any teams run by your school or community groups)." The 4 response options were $0,1,2$, or 3 or more teams. Students were classified as participating in sports if they selected a response option of 1 or more teams. ${ }^{6}$

Youth Attending Daily PE Classes. To assess students attending daily PE classes, students were asked, "In an average week when you are in school, on how many days do you go to physical education (PE) classes?" The 6 response options were 0 to 5 days. Students were classified as attending daily PE if they selected "5 days."6

\section{Demographic Characteristics}

Student demographic characteristics included in the analyses were sex (male or female), grade $(9,10,11$, or 12$)$, and race/ethnicity. Race/ethnicity was categorized into White, non-Hispanic (White); Black or African American, non-Hispanic (Black); Hispanic or Latino (Hispanic); and Other. The racial/ethnic group "Other" will not be interpreted because of the heterogeneity of the group, which includes students of non-Hispanic racial groups other than White and Black, as well as students of 2 or more races.

\section{Statistical Analysis}

Data for each year were stratified by sex and then analyzed by grade and race/ethnicity to estimate the prevalence and $95 \%$ confidence intervals for all 5 physical activity behaviors. Sex-stratified logistic regression models with a year main effect were developed (model $1)$, then sex-stratified logistic regression models with a year by grade interaction term (model 2) and a year by race/ethnicity interaction term (model 3) were developed. For each sex-stratified model, linear and quadratic trends over time were analyzed overall, by grade, and by racial/ethnic subgroup. Significant quadratic trends without a significant linear trend will not be interpreted, as they represent no significant linear change across the period. 
Tests of differences from 2009 or 2011 to 2019 (change over time) overall, for each grade, and for each racial/ethnic group were also incorporated into the sex-specific logistic regression models. Tests of differences in differences (ie, differences between these sex-stratified groups' and subgroups' differences from 2009 or 2011 to 2019) were also incorporated into the models. For example, the change from 2009 or 2011 to 2019 in a physical activity behavior for ninth-grade males could be significantly different than the change from 2009 or 2011 to 2019 for 10th-grade males, or there could be a significant difference in differences between White females and Black females. Significance level was $P<.05$ for trends and single comparisons. Bonferroni adjustments were used within each category for multiple comparisons, to $P<.025$ for 2 tests, $P<.0125$ for 4 tests, or $P<.0083$ for 6 tests. For example, change over time tests for males and females were adjusted to $P<.025$, whereas change over time tests for the 4 sex-stratified grade groups and 4 sex-stratified race/ethnicity groups were adjusted to $P<.0125$, and sex-stratified differences of differences tests by grade and race/ethnicity were adjusted to $P<.0083$. Analyses were conducted using SUDAAN (version 11.0; Research Triangle Institute, Research Triangle Park, NC) to account for survey design and weights.

\section{Results}

From 2009 to 2019, the weighted sample distribution was nearly evenly split by sex, with $50.7 \%$ males and $49.3 \%$ females overall (Table 1). Distribution by sex-stratified grade was similar across all years, whereas distribution by sex-stratified race/ethnicity varied somewhat by year (see Supplementary Table S1 [available online]). About a quarter of the students were in each high school grade level, ranging from a slightly larger portion of students in ninth grade to a smaller portion of students in 12 th grade. Over half were White, one-fifth were Hispanic, and the remaining students were Black and Other races/ethnicities.

\section{Physical Activity Guidelines}

In $2019,31.0 \%$ of males and $15.4 \%$ of females met the aerobic guideline, $59.0 \%$ of males and $39.8 \%$ of females met the musclestrengthening guideline, and $23.1 \%$ of males and $10.2 \%$ of females met both (Table 2). Among males overall, examination of the absolute difference from 2011 to 2019 yielded significant decreases in all 3 guidelines-based behaviors (range: -7.6 to -7.2 percentage points, $P<.025)$. These decreases were also seen for most male subgroups by grade and race/ethnicity (range: -14.9 to -5.2 percentage points, $P<.0125)$. Among females overall, there were also significant absolute declines in all guidelines-based behaviors (range: -4.1 to -2.6 percentage points, $P<.025$ ) from 2011 to 2019. Among female subgroups, there was only one significant absolute decline of 3.6 percentage points $(19.7 \%$ $16.0 \%, P<.0125)$ among White females meeting the aerobic guideline. Differences in differences for guidelines-based behaviors between overall males and overall females were significant $(P<.05)$; however, no significant differences in differences were observed among sex-stratified subgroups.

From 2011 to 2019 , the prevalence of meeting the aerobic, muscle-strengthening, and both guidelines also showed decreasing trends among males overall and across most male subgroups by grade and race/ethnicity (Table 2; Figures 1-3). Among females, decreasing trends were observed overall and among 10th graders across all 3 guidelines-based behaviors (Figures 1A-3A). Decreasing trends by racial/ethnic groups were observed among White, Black, and Other females for meeting the aerobic guideline and among White and Black females for meeting both guidelines (Figures 1B and 3B).

Table 1 Sex-Stratified Participant Characteristics, All Years Combined, YRBS 2009-2019

\begin{tabular}{|c|c|c|c|c|}
\hline \multirow[b]{2}{*}{ Characteristics } & \multicolumn{2}{|c|}{ Male } & \multicolumn{2}{|c|}{ Female } \\
\hline & $\mathbf{n}$ & $\%(95 \% \mathrm{Cl})^{\mathrm{a}}$ & $\mathbf{n}$ & $\%(95 \% \mathrm{Cl})^{\mathrm{a}}$ \\
\hline Total & 42,965 & $50.7(49.8-51.6)$ & 43,904 & $49.3(48.4-50.2)$ \\
\hline \multicolumn{5}{|l|}{ Year } \\
\hline 2009 & 7888 & $52.1(49.7-54.6)$ & 8153 & $47.9(45.4-50.3)$ \\
\hline 2011 & 7469 & $51.4(49.6-53.2)$ & 7557 & $48.6(46.8-50.4)$ \\
\hline 2013 & 6739 & $49.8(48.5-51.0)$ & 6485 & $50.2(49.0-51.5)$ \\
\hline 2015 & 7530 & $51.0(47.7-54.3)$ & 7599 & $49.0(45.7-52.3)$ \\
\hline 2017 & 6933 & $49.1(46.6-51.6)$ & 7395 & $50.9(48.4-53.4)$ \\
\hline 2019 & 6406 & $50.4(48.9-52.0)$ & 6715 & $49.6(48.0-51.1)$ \\
\hline \multicolumn{5}{|l|}{ Grade } \\
\hline Ninth grade & 10,959 & $27.7(26.9-28.5)$ & 11,526 & $26.8(26.1-27.6)$ \\
\hline 10th grade & 10,709 & $25.8(25.0-26.7)$ & 10,960 & $25.7(25.1-26.3)$ \\
\hline 11 th grade & 10,959 & $23.8(23.3-24.3)$ & 10,865 & $24.1(23.5-24.6)$ \\
\hline 12th grade & 10,338 & $22.7(22.1-23.3)$ & 10,553 & $23.4(22.8-24.0)$ \\
\hline \multicolumn{5}{|l|}{ Race/ethnicity } \\
\hline White, NH & 19,001 & $55.5(53.0-58.0)$ & 19,128 & $55.0(52.6-57.4)$ \\
\hline Black or AA, NH & 7364 & $13.7(12.5-15.0)$ & 7652 & $13.7(12.6-14.9)$ \\
\hline Hispanic or Latino & 11,989 & $21.5(19.8-23.2)$ & 12,364 & $21.8(20.2-23.5)$ \\
\hline Other & 4611 & $9.3(8.4-10.3)$ & 4760 & $9.5(8.5-10.6)$ \\
\hline
\end{tabular}

Abbreviations: AA, African American; CI, confidence interval; NH, non-Hispanic; YRBS, Youth Risk Behavior Survey.

${ }^{a}$ Weighted percentage. 


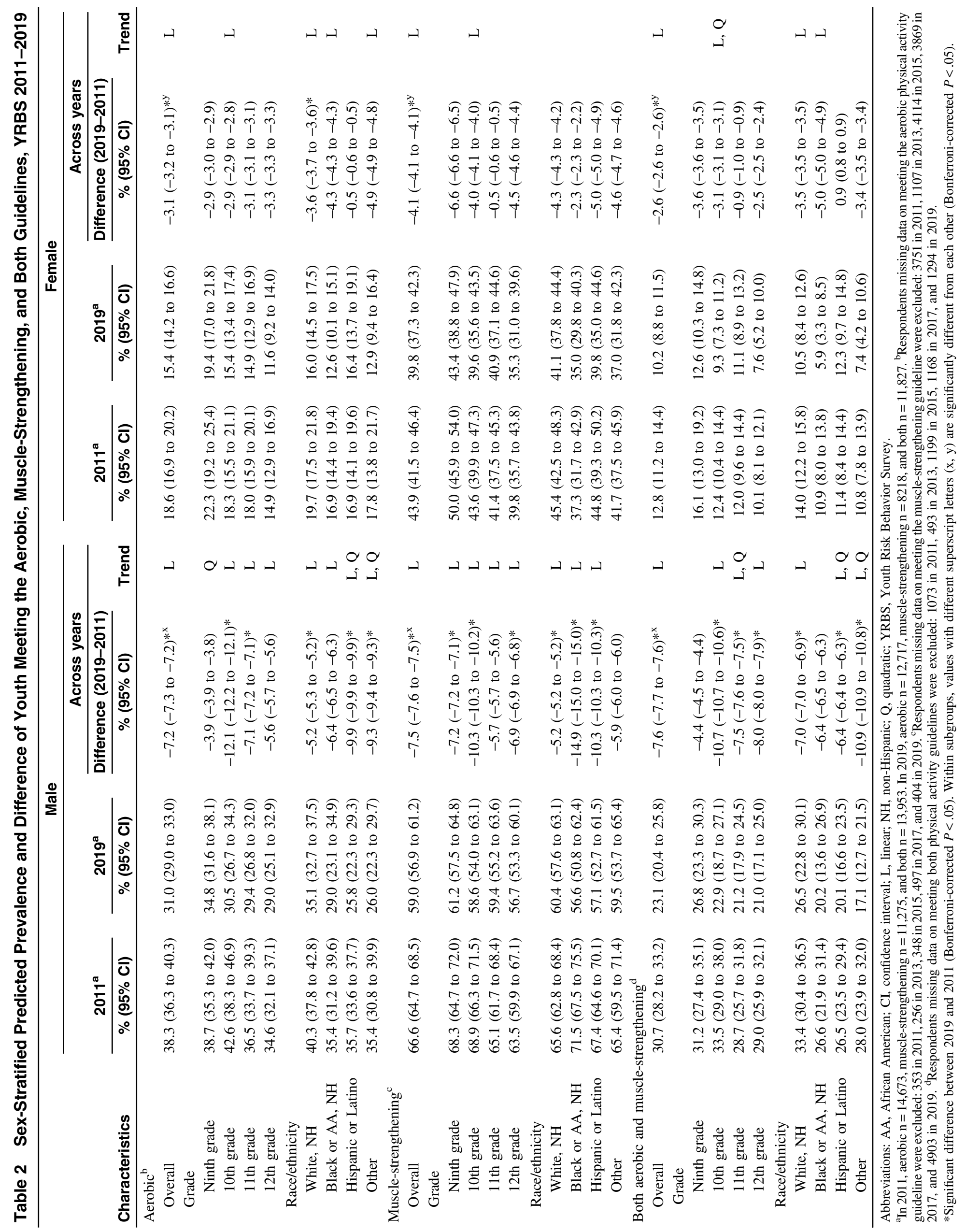


A

50

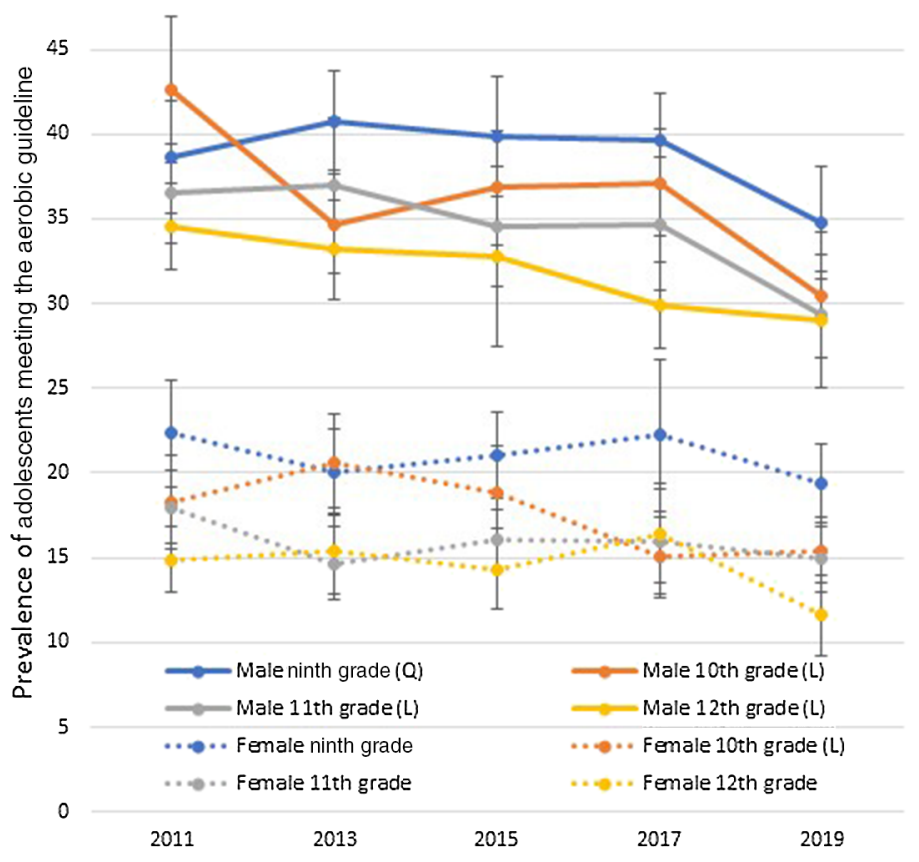

B

50

45

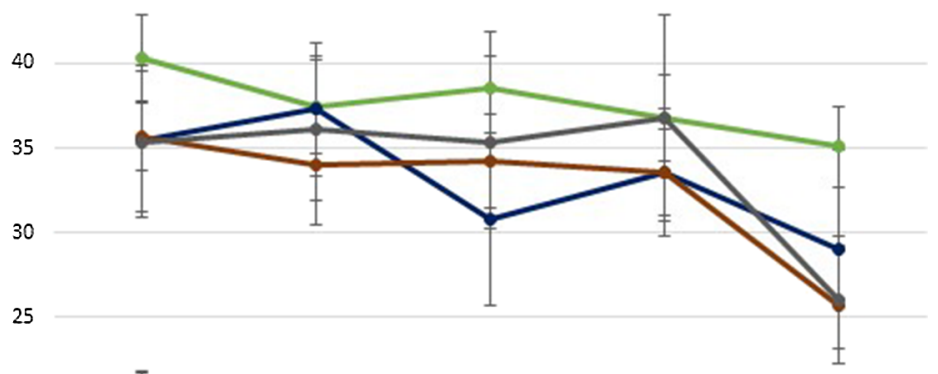

20

15

10

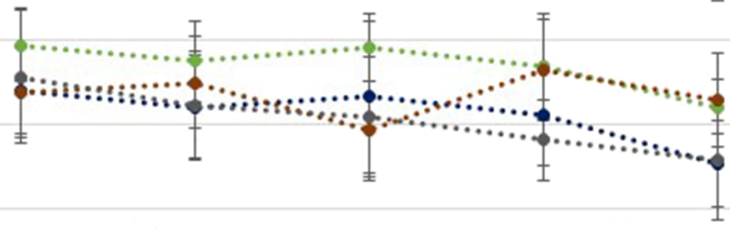

$\longrightarrow$ Male White $(L) \quad$ Male Black (L)

$5 \longrightarrow$ Male Hispanic/Latino (L, Q) $\longrightarrow$ Male Other (L, Q)

..... Female White $\quad$..... Female Black

..... Female Hspanic/Latino

$\begin{array}{lllll}2011 & 2013 & 2015 & 2017 & 2019\end{array}$

Figure 1 - Sex-stratified trends by grade and race/ethnicity in predicted prevalence of youth meeting the aerobic guideline, YRBS 2011-2019. Error bars represent the lower and upper bounds of the 95\% CI. CI indicates confidence interval; L, linear trend; L, Q, both L and Q trends; Q, quadratic trend; YRBS, Youth Risk Behavior Survey.

A

Males and females, by grade

80

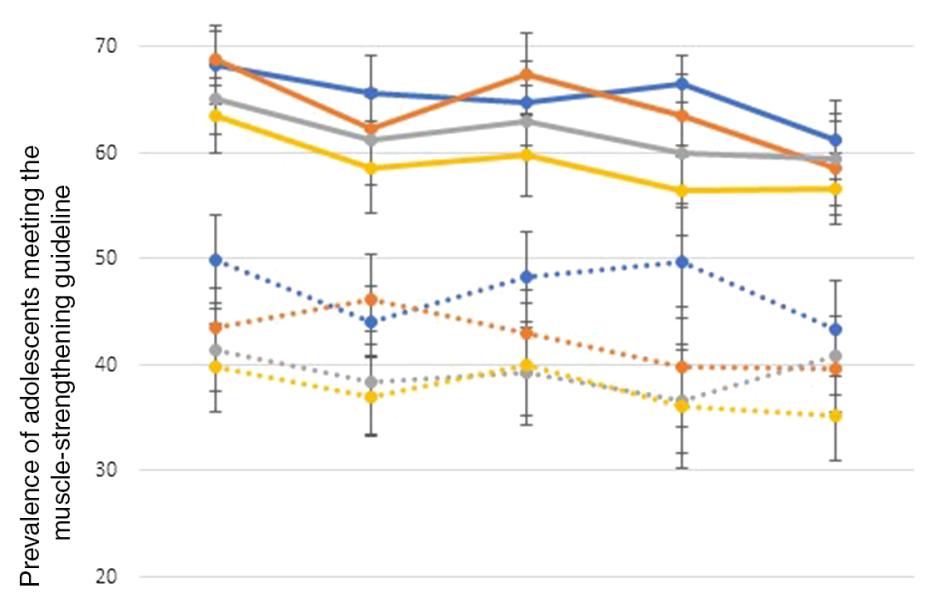

B

80

70

60

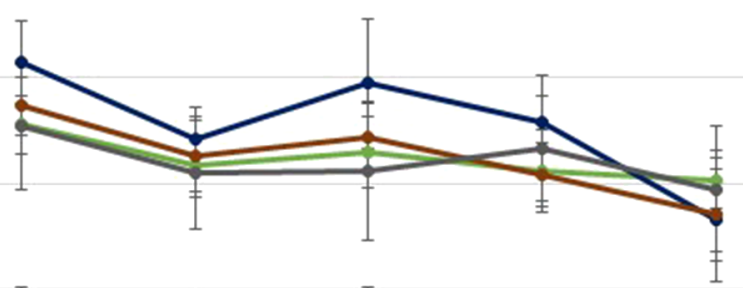

50

40

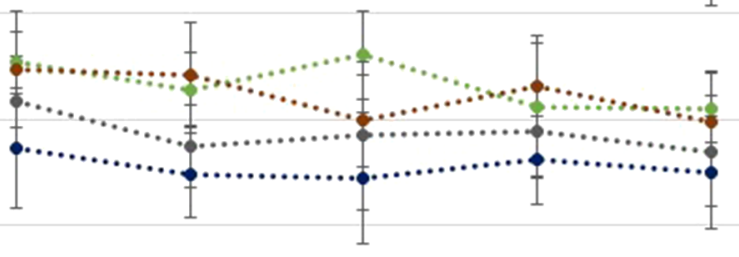

20

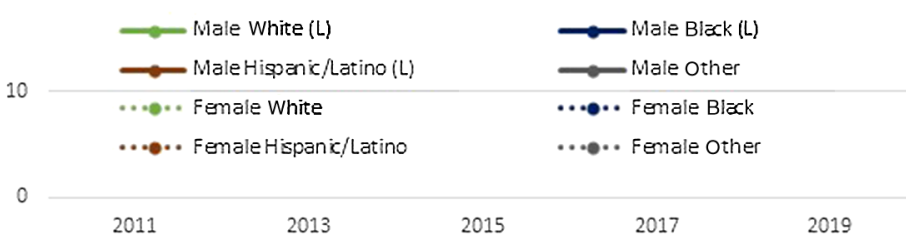

Figure 2 - Sex-stratified trends by grade and race/ethnicity in predicted prevalence of youth meeting the muscle-strengthening guideline, YRBS 2011-2019. Error bars represent the lower and upper bounds of the 95\% CI. CI indicates confidence interval; L, linear trend; L, Q, both L and Q trends; Q, quadratic trend; YRBS, Youth Risk Behavior Survey. 
A

40

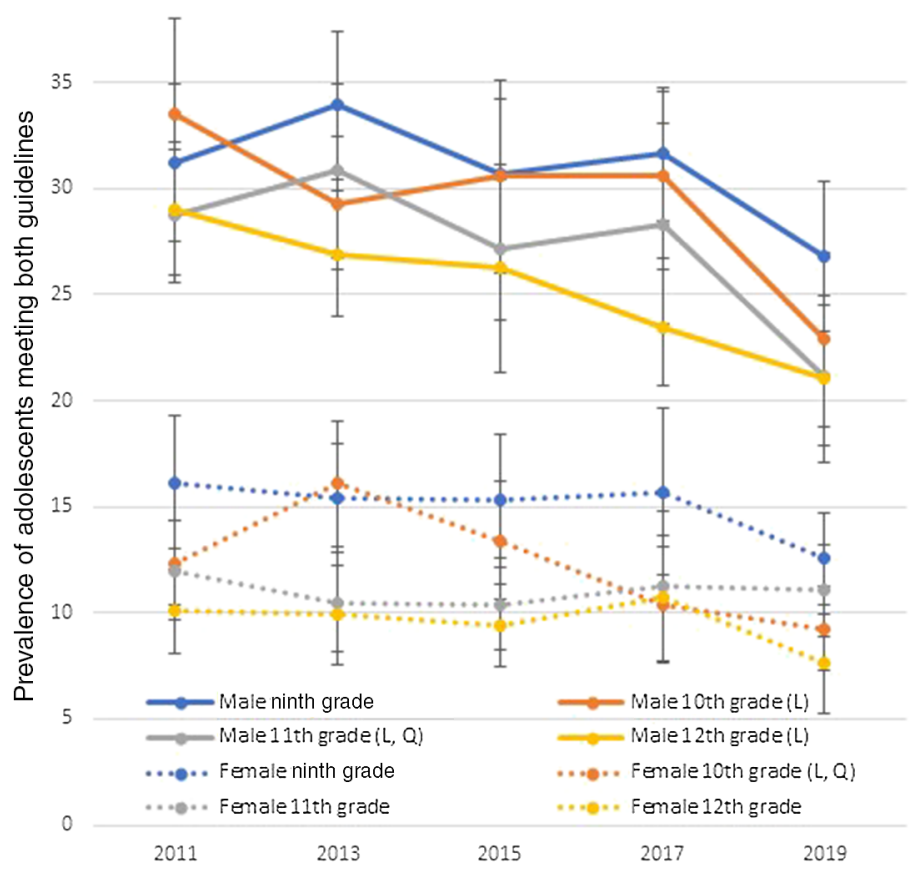

B

40

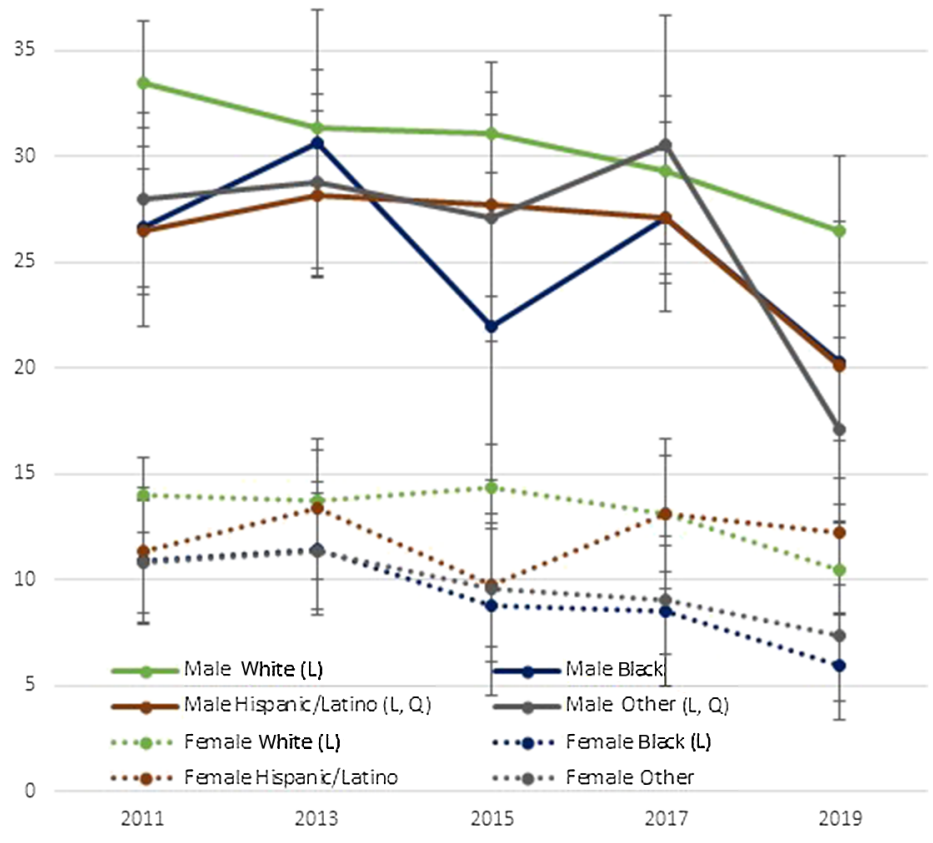

Figure 3 - Sex-stratified trends by grade and race/ethnicity in predicted prevalence of youth meeting both aerobic and muscle-strengthening guidelines, YRBS 2011-2019. Error bars represent the lower and upper bounds of the 95\% CI. CI indicates confidence interval; L, linear trend; L, Q, both L and Q trends; Q, quadratic trend; YRBS, Youth Risk Behavior Survey.

\section{Sports Participation and Daily PE Attendance}

In $2019,60.4 \%$ of males and $54.7 \%$ of females participated in sports, with neither overall males nor overall females displaying significant absolute differences or trends from 2009 to 2019 (Table 3). Of all subgroups, male and female, only Hispanic males had a significant absolute 7.1 percentage point decrease $(62.0 \%-$ $54.8 \%, P<.0125)$ in sports participation. The difference in differences between overall males and overall females was significant $(-5.8$ percentage points, $P<.05)$; no significant differences in differences were observed among sex-stratified subgroups. From 2009 to 2019, among male subgroups, there was a decreasing trend among 10th-grade males (Figure 4A) and Hispanic males (Figure 4B). Among female subgroups, there were significant quadratic trends (ie, nonlinear decrease then increase) in sports participation for ninth-grade and 11th-grade females.

In $2019,29.2 \%$ of males and $22.8 \%$ of females attended daily PE, with overall females but not overall males displaying a significant absolute difference $(-9.2$ percentage points, $P<.025)$ and decreasing trend from 2009 to 2019 (Table 3). Most male subgroups exhibited no significant absolute differences, except for a significant absolute decline of 14.7 percentage points $(40.2 \%-$ $25.5 \%, P<.0125)$ in attending daily PE among Black males (Table 3). Among female subgroups, from 2009 to 2019, there were significant absolute differences $(P<.0125)$ among ninth graders and Hispanic females of 15.6 percentage points $(48.7 \%-$ $33.0 \%$ ) and 12.5 percentage points $(39.5 \%-27.1 \%)$, respectively. No significant differences in differences were observed overall or among sex-stratified subgroups. Similar to absolute differences among male subgroups, only Black males showed a decreasing trend in daily PE (Figure 5B). Among female subgroups, decreasing trends were found for ninth-grade, 10th-grade, and White females; a significant quadratic trend (ie, nonlinear decrease then increase) was found for 11th-grade females (Figure 5A and $5 \mathrm{~B})$.

\section{Discussion}

Decreases in prevalence of meeting physical activity guidelines were ubiquitous among nearly all high school male subgroups by grade and race/ethnicity from 2011 to 2019 , whereas high school female subgroups exhibited either a decline or no significant change, which could be explained, in part, by the already low prevalence of high school females meeting the guidelines. In addition to these patterns for meeting guidelines, the strategies of sports participation and daily PE to increase youth physical activity showed relatively few sex-specific subgroup changes during 2009-2019. These findings show a need for concurrent efforts on broad interventions appropriate for all adolescents and tailored interventions for sex-specific subgroups of adolescents by grade and race/ethnicity as important ways for increasing youth physical activity, including but not limited to the school and youth program strategies of sports participation and daily PE.

\section{Patterns and Disparities During the Past Decade}

Summaries of the national YRBS have previously detailed adolescent youth physical activity behaviors in the United States. Overall trends and changes between adjacent survey years were examined from 2011 to 2017, ${ }^{16-19}$ and in 2019, trends and 2017-2019 changes were examined by individual sex and racial/ethnic 


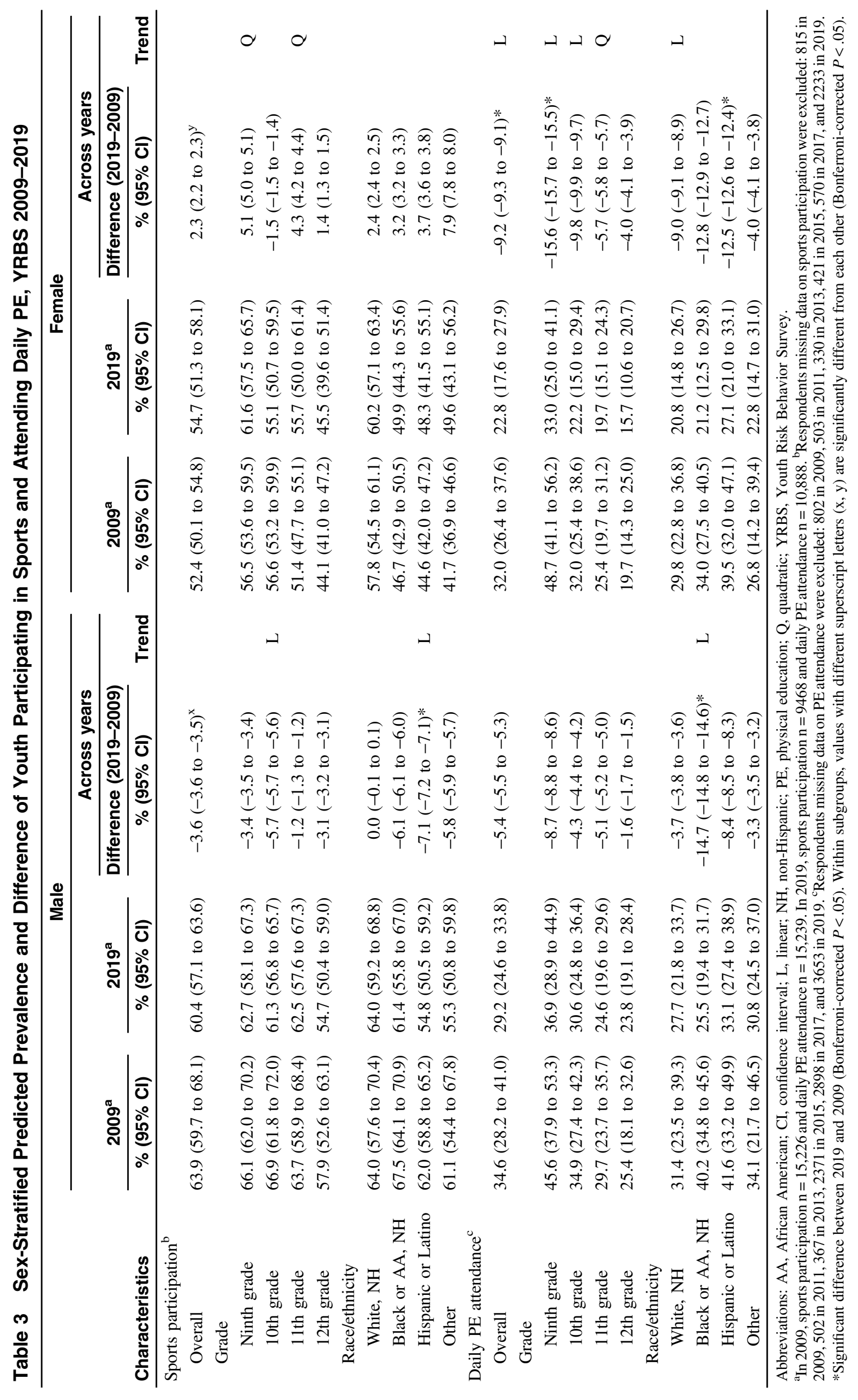


A

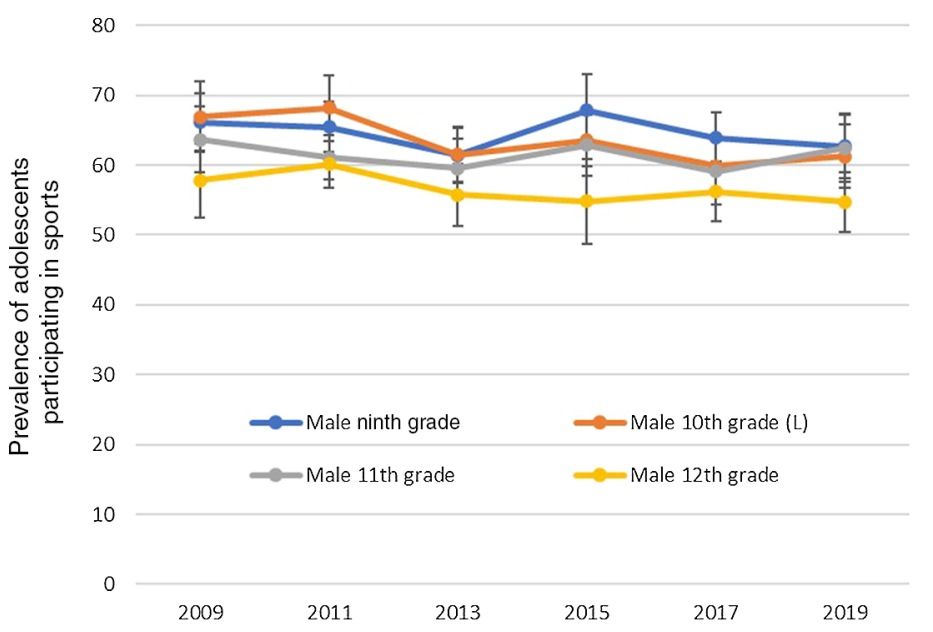

B

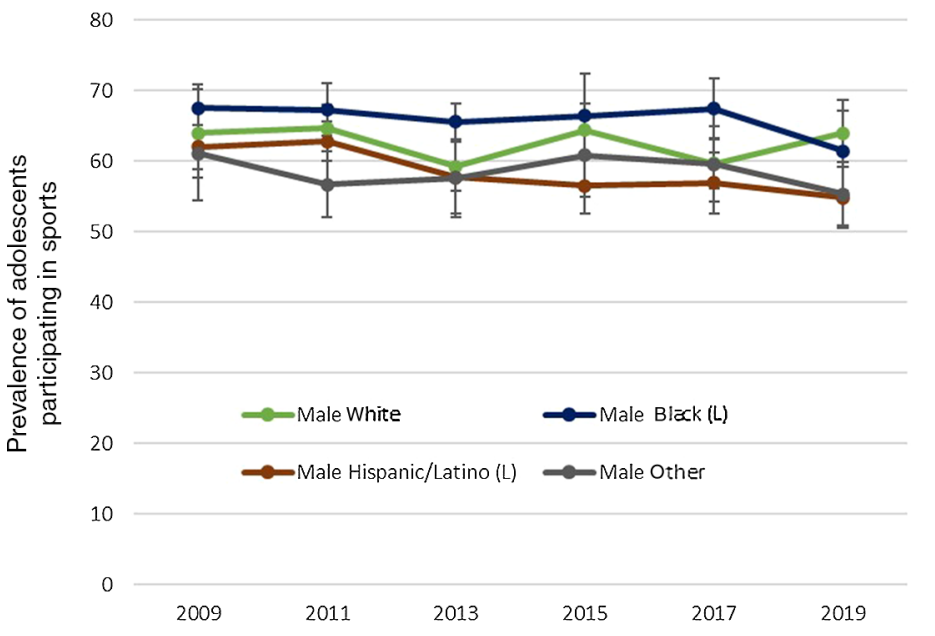

Females, by grade

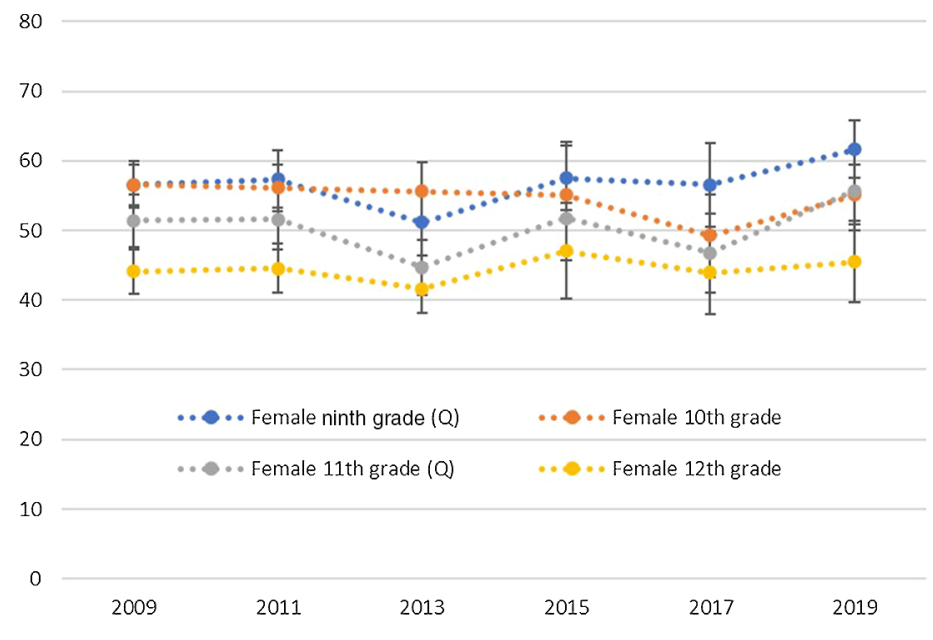

Females, by race/ethnicity

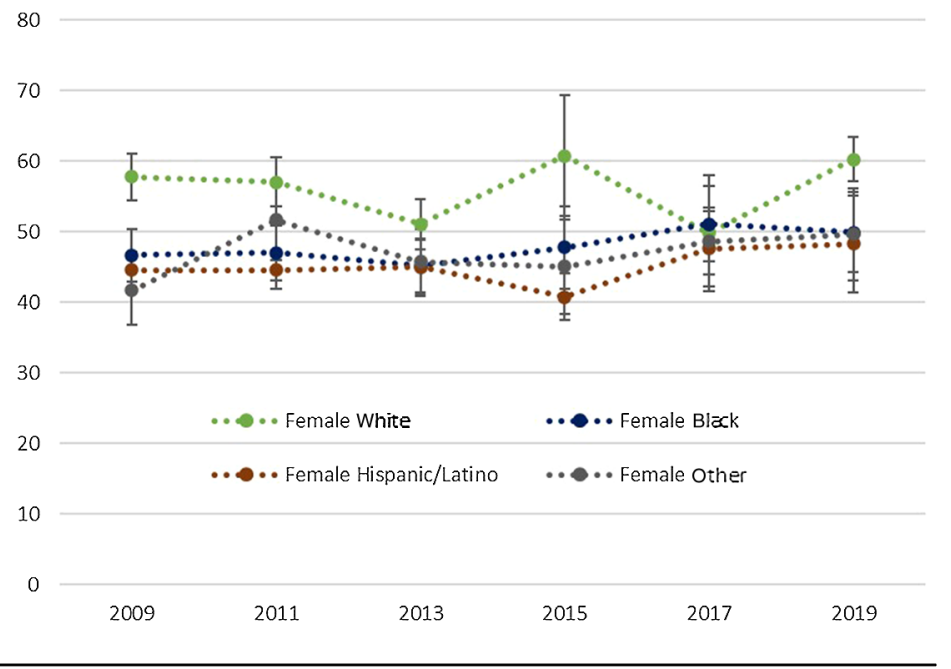

Figure 4 - Sex-stratified trends by grade and race/ethnicity in predicted prevalence of youth participating in sports, YRBS 2009-2019. Error bars represent the lower and upper bounds of the 95\% CI. CI indicates confidence interval; L, linear trend; L, Q, both L and Q trends; Q, quadratic trend; YRBS, Youth Risk Behavior Survey.

groups. ${ }^{9}$ From 2011 through 2017, prevalence of all youth meeting the aerobic guideline, meeting the muscle-strengthening guideline, participating in at least one sports team, and attending daily PE mostly had no significant changes, other than 2011-2013 decreases in meeting the muscle-strengthening guideline and participating in sports. ${ }^{16-19}$ A report that added the 2019 YRBS data found more significant linear decreases among all youth meeting the aerobic, muscle-strengthening, and both guidelines during 2011-2019.9 Moreover, male, White, and Black youth showed significant linear decreases in meeting the aerobic, muscle-strengthening, and both guidelines; Hispanic youth showed significant linear decreases in meeting the aerobic guideline and muscle-strengthening guideline; and female youth showed significant linear decreases in meeting the aerobic guideline, meeting both guidelines, and attending daily PE (2009-2019). ${ }^{9}$ Our study extends these findings by identifying more nuanced patterns from sex-stratified estimates by grade and race/ethnicity, which could not be observed previously among the broader group trends.

Although our study's trends correspond with those by individual sex and racial/ethnic groups, we discovered grade and racial/ ethnic subgroup physical activity behavior changes that differed by sex. For example, Hispanic males, but not Hispanic females, showed decreasing trends and differences in meeting the aerobic, muscle-strengthening, and both guidelines, as well as in sports participation; ninth-grade females, but not ninth-grade males, showed a decreasing trend and difference in daily PE attendance. In addition, Black males showed a decreasing trend and difference in daily PE attendance that their Black female counterparts did not. These specific changes in adolescent physical activity are valuable additions for public health and education, as they can be used to gauge progress among subgroups of youth and to guide ongoing decisions for increasing youth physical activity, including using strategies beyond sports and PE with these subgroups.

There is no comparable youth physical activity research for the past decade on possible differences in sex-stratified changes over time by grade and race/ethnicity, so these new findings may highlight subgroups experiencing disparities. Early surveillance from 1999 to 2005 YRBS yielded a decrease in ninth-grade males participating in sufficient moderate physical activity, but increases in Hispanic females participating in sufficient moderate physical 
A

Males, by grade

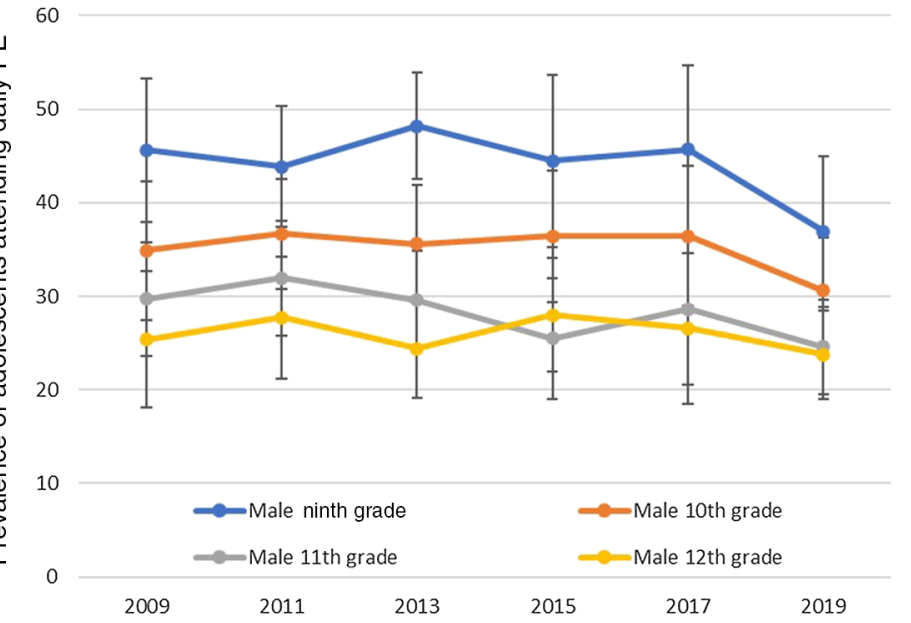

B

Males, by race/ethnicity

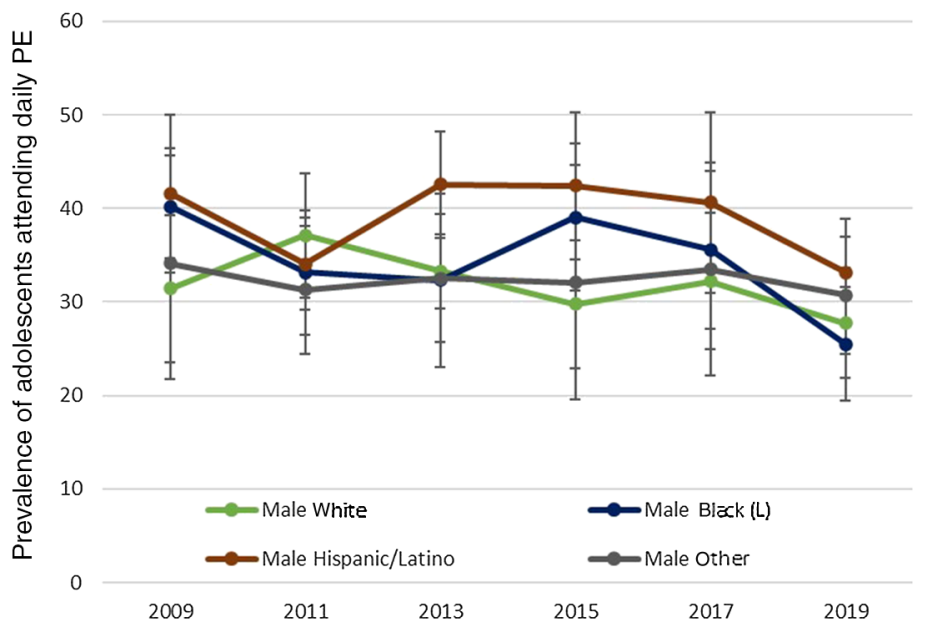

Females, by grade

60

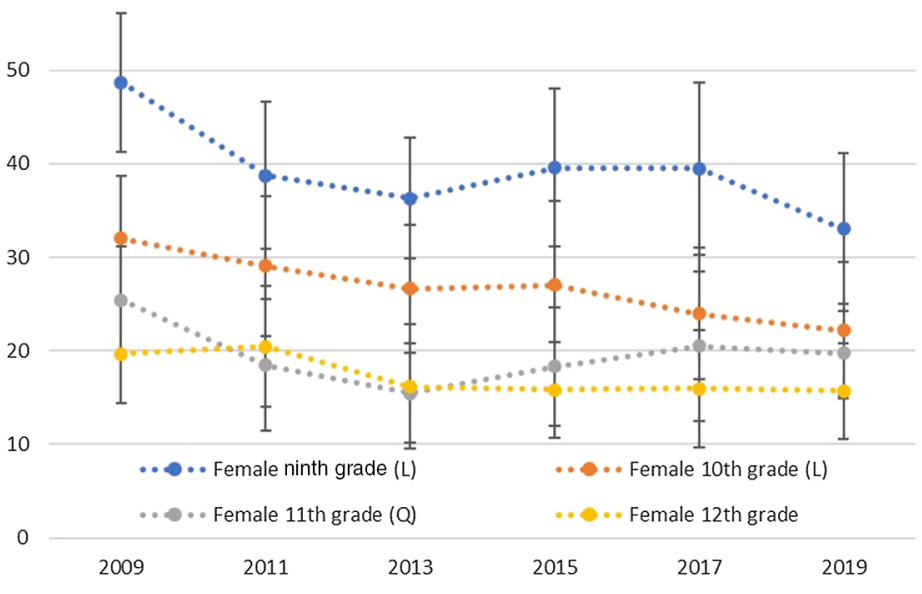

Females, by race/ethnicity

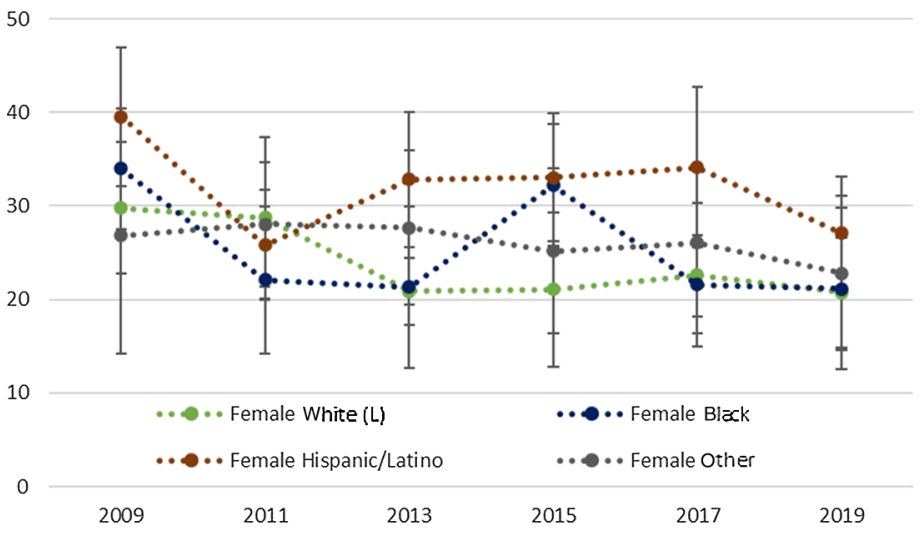

Figure 5 - Sex-stratified trends by grade and race/ethnicity in predicted prevalence of youth attending daily PE, YRBS 2009-2019. Error bars represent the lower and upper bounds of the 95\% CI. CI indicates confidence interval; L, linear trend; L, Q, both L and Q trends; PE, physical education; Q, quadratic trend; YRBS, Youth Risk Behavior Survey.

activity. ${ }^{23}$ In addition, there were increases in 10th-grade females participating in no moderate- to vigorous-intensity physical activity during the same period. ${ }^{23}$ Although these earlier findings are from a different time period with different assessment of physical activity behaviors, they support continued focus on sex-specific subgroup trends, which may indicate changing disparities. In the current study, males overall and females overall had declines from 2009 or 2011 to 2019 for all behaviors, except for a nonsignificant increase in overall females' sports participation. For all guidelines-based behaviors and sports participation, these differences in males overall were significantly different than for females overall (ie, significant overall differences in differences), indicating the lessening of overall sex disparities. However, this lessening is a result of worsening patterns among male adolescents and unimproved patterns among female adolescents. Regardless of changes over this time, disparities between grade and racial/ethnic groups remained the same (ie, no significant subgroup differences in differences). Notably, however, all significant differences were decreases in physical activity.

\section{Strategies and Factors Influencing Physical Activity Patterns}

While more widespread declines were seen for guidelines-based behaviors especially among male adolescents by grade and race/ ethnicity, there were moderately few decreasing sex-specific subgroup trends and differences for sports participation and daily PE attendance, resulting in mostly steady patterns during the past decade. Data on the amount of physical activity gained through sports and PE support these observed differences when compared with guidelines-based behaviors-participating in sports or PE does not necessarily lead to meeting the physical activity guidelines. Although observed among a younger age range, one study found that only $24 \%$ of youth aged 7-14 years met the recommended 60 minutes through sports practice overall, with more younger than older youth and boys than girls getting significantly more MVPA. ${ }^{24}$ Though PE policies and offerings for students vary, mandatory daily PE was estimated to provide an average of 23 minutes of MVPA per school day, ${ }^{25}$ or about $40 \%$ of the recommended 60 minutes on 5 of 
the 7 days youth need to be active every week. These studies may help explain why the declines of youth meeting the guidelines are not necessarily reflected by the patterns seen for sports and PE. Moreover, due to barriers such as school closures and restricted peer interactions in 2020, ${ }^{26-29}$ further decreases across youth physical activity measures suggest that not only are safe school-based efforts $^{30}$ like those in the Comprehensive School Physical Activity Program Framework ${ }^{31}$ and efforts outside the school setting like those in the National Youth Sports Strategy ${ }^{4}$ needed, additional novel strategies beyond sports and PE may be beneficial or even crucial in helping youth meet the physical activity guidelines.

Though availability of inclusive, accessible, and engaging sports opportunities and access to effective, comprehensive PE programs differs, these strategies can substantially help youth meet guidelines. ${ }^{4,25}$ Because the quality and availability of sports and PE opportunities often depends on policy and program decisions, some factors affecting participation may be different than those influencing other, more general, physical activity behaviors. For example, barriers to entering or continuing youth sports, for which programs could provide adaptations, include the following: lack of access, cost, social or physical factors, and stress or burnout (eg, from being overly competitive or physically intense). ${ }^{4}$ School policies, such as mandatory PE for all youth, standardized PE curricula, and/or accountability in PE instruction and assessment, may depend on policymakers passing legislative mandates that consider any exemptions, compliance monitoring, or enforcement, ${ }^{25}$ or require the initiative and collaboration of school leaders and practitioners to ensure effective and sustainable PE policy environments, curricula, instruction, and assessment for students. ${ }^{32,33}$ To illustrate the impact that policy and program change can have on physical activity levels among high-need groups, the Physical Activity Guidelines for Americans Midcourse Report detailed how title IX expanded physical activity opportunities for millions of girls in 1972. ${ }^{3}$ By targeting gender inequity with bans against gender-based exclusion from educational programs and activities, title IX encouraged a more than tenfold increase over 40 years in sports participation among high school girls. ${ }^{3}$ By addressing barriers for both male and female adolescents, broad policies and programs influencing sports and PE could, in turn, affect sex-specific subgroups.

In explaining the current findings, it is also important to consider the various factors that affect youth physical activity behaviors, ranging from individual characteristics and interpersonal support to community-level and environmental correlates. ${ }^{26,34}$ These factors likely contribute to our findings of trends and decreases and add complexity in addressing these behaviors; many of these factors are addressed in the Active People, Healthy Nation ${ }^{\mathrm{SM}}$ strategies to increase physical activity, such as community and environmental strategies like ActivityFriendly Routes to Everyday Destinations and Access to Places for Physical Activity. ${ }^{5}$ In addition, factors may overlap, as in a study of focus groups, in which African American and Latino participants named several barriers: lack of intrapersonal motivation or time, social support, and environmental safety concerns. ${ }^{35}$ In a longitudinal study of physical activity from childhood to adolescence, declines were observed in self-efficacy alongside greater perceptions of barriers, greater declines in enjoyment or fitness goals, or smaller declines in appearance or social goals. ${ }^{36}$ Specific correlates differ by intersectional identities of race/ethnicity and sex, ${ }^{37}$ such as the significant association of physical activity levels with family support in a church-based intervention for Black adolescent females. ${ }^{38}$ Thus, because factors of particular importance to sexstratified subgroups by grade and race/ethnicity can be useful levers for tailored interventions, more research would be beneficial to explore specific interventions suitable for these subgroups.

\section{Limitations}

This study has a few limitations. First, YRBS survey data are selfreported and may be subject to biases (eg, recall and social desirability) leading to an unknown amount of underreporting or overreporting. ${ }^{15-19}$ For example, both underestimates and overestimates occurred when concurrent validity was tested by comparing YRBS self-report measures to accelerometry data, varying by activity intensity level and duration (eg, accumulated vs bouts) ${ }^{39}$; the MVPA measure showed reasonable validity. ${ }^{40}$ Second, the amount of missing data (ie, more than $5 \%$ ) for some of the behaviors and years analyzed in this study (eg, muscle-strengthening for 20112019) was a limitation; missing data were excluded from the analysis. If youth with missing data for these behaviors and years were systematically more or less physically active than youth included in the analysis, then our study's results could be inaccurate representations of the past decade of youth physical activity. Third, our study focused on differences and trends of youth physical activity by subgroups, but we did not compare the prevalence of youth physical activity by subgroup in individual years. However, multiple other studies have reported these cross-sectional comparisons; they have found higher prevalence among male versus females, younger grades versus older grades, males of each grade level and racial/ethnic group versus their female counterparts, ${ }^{15-19}$ and White versus their Black and Hispanic counterparts for multiple physical activity behaviors. ${ }^{9}$ Finally, the adolescent physical activity measures presented are only those available through YRBS data; beyond aerobic activity, muscle-strengthening activity, sports, and $\mathrm{PE}$, other guidelines components ${ }^{2}$ and strategies ${ }^{3}$ may be similarly important for youth physical activity.

\section{Conclusions}

This study's sex-stratified trends and differences by grade and race/ ethnicity describe the following: decreases across almost all male adolescent subgroups meeting the physical activity guidelines, and subgroup declines or no change in the low prevalence of female adolescents meeting guidelines. For the school and youth program strategies of sports and PE, relatively few subgroup changes were observed. Concurrent efforts for both broad strategies for all adolescents and tailored interventions for sex-specific subgroups, to supplement sports and PE, could help increase youth physical activity as an important health behavior.

\section{Acknowledgments}

The findings and conclusions in this report are those of the authors and do not necessarily represent the official position of the Centers for Disease Control and Prevention. The authors declared no potential conflicts of interest with respect to the research, authorship, and/or publication of this article. The authors received no financial support for the research, authorship, and/or publication of this article.

\section{References}

1. US Department of Health and Human Services. 2008 Physical Activity Guidelines for Americans. Washington, DC: US Department of Health and Human Services; 2008. 
2. US Department of Health and Human Services. Physical Activity Guidelines for Americans, 2nd edition. Washington, DC: US Department of Health and Human Services; 2018.

3. Physical Activity Guidelines for Americans Midcourse Report Subcommittee of the President's Council on Fitness, Sports \& Nutrition. Physical Activity Guidelines for Americans Midcourse Report: Strategies to Increase Physical Activity Among Youth. Washington, DC: US Department of Health and Human Services; 2012.

4. US Department of Health and Human Services. National Youth Sports Strategy. Washington, DC: US Department of Health and Human Services; 2019.

5. Schmid TL, Fulton JE, McMahon JM, Devlin HM, Rose KM, Petersen R. Delivering physical activity strategies that work: Active People, Healthy NationSM. J Phys Act Health. 2021;18(4):352-356. PubMed ID: 33639612 doi:10.1123/jpah.2020-0656

6. US Department of Health and Human Services, Office of Disease Prevention and Health Promotion. Physical Activity-Healthy People 2030. 2020. https://health.gov/healthypeople/objectives-anddata/browse-objectives/physical-activity. Accessed March 1, 2021.

7. Belcher BR, Berrigan D, Dodd KW, Emken BA, Chou CP, SpruijtMetz D. Physical activity in US youth: effect of race/ethnicity, age, gender, and weight status. Med Sci Sports Exerc. 2010;42(12):22112221. PubMed ID: 21084930 doi:10.1249/MSS.0b013e3181e1fba9

8. Gordon-Larsen P, McMurray RG, Popkin BM. Adolescent physical activity and inactivity vary by ethnicity: the National Longitudinal Study of Adolescent Health. J Pediatr. 1999;135(3):301-306. PubMed ID: 10484793 doi:10.1016/S0022-3476(99)70124-1

9. Merlo CL, Jones SE, Michael SL, et al. Dietary and physical activity behaviors among high school students-Youth Risk Behavior Survey, United States, 2019. MMWR Suppl. 2020;69(1):64-76. PubMed ID: 32817612 doi:10.15585/mmwr.su6901a8

10. Troiano RP, Berrigan D, Dodd KW, Masse LC, Tilert T, McDowell M. Physical activity in the United States measured by accelerometer. Med Sci Sports Exerc. 2007;40(1):181-188. doi:10.1249/mss.0b013e 31815a51b3

11. Spencer RA, Rehman L, Kirk SF. Understanding gender norms, nutrition, and physical activity in adolescent girls: a scoping review. Int J Behav Nutr Phys Act. 2015;12(1):6. PubMed ID: 25616739 doi:10.1186/s12966-015-0166-8

12. Cairney J, Veldhuizen S, Kwan M, Hay J, Faught BE. Biological age and sex-related declines in physical activity during adolescence. Med Sci Sports Exerc. 2014;46(4):730-735. PubMed ID: 24056271 doi:10.1249/MSS.0000000000000168

13. Caspersen CJ, Pereira MA, Curran KM. Changes in physical activity patterns in the United States, by sex and cross-sectional age. Med Sci Sports Exerc. 2000;32(9):1601-1609. PubMed ID: 10994912 doi:10. 1097/00005768-200009000-00013

14. Colabianchi N, Griffin JL, Slater SJ, O’Malley PM, Johnston LD. The whole-of-school approach to physical activity: findings from a national sample of US secondary students. Am J Prev Med. 2015;49(3):387394. PubMed ID: 26188684 doi:10.1016/j.amepre.2015.02.012

15. Eaton DK, Kann L, Kinchen S, et al. Youth Risk Behavior Surveillance-United States, 2009. MMWR Surveill Summ. 2010;59(5):1142. PubMed ID: 20520591

16. Eaton DK, Kann L, Kinchen S, et al. Youth Risk Behavior Surveillance-United States, 2011. MMWR Surveill Summ. 2012;61(4):1162. PubMed ID: 22673000

17. Kann L, Kinchen S, Shanklin SL, et al. Youth Risk Behavior Surveillance-United States, 2013. MMWR Surveill Summ. 2014;63(4):1-168.

18. Kann L, McManus T, Harris WA, et al. Youth Risk Behavior Surveillance-United States, 2015. MMWR Surveill Summ. 2016;65(6):1-174. PubMed ID: 27280474 doi:10.15585/mmwr.ss6506a1
19. Kann L, McManus T, Harris WA, et al. Youth Risk Behavior Surveillance-United States, 2017. MMWR Surveill Summ. 2018;67(8):1-114. PubMed ID: 29902162 doi:10.15585/mmwr.ss6708a1

20. Lowry R, Lee SM, Fulton JE, Demissie Z, Kann L. Obesity and other correlates of physical activity and sedentary behaviors among US high school students. J Obes. 2013;2013:1-10. doi:10.1155/2013/276318

21. Miller J, Pereira M, Wolfson J, Laska M, Nelson T, NeumarkSztainer D. Developmental trends and determinants of physical activity from adolescence to adulthood differ by ethnicity/race and sex. J Phys Act Health. 2018;15(5):345-354. PubMed ID: 29421966 doi:10.1123/jpah.2017-0287

22. Watson KB, Harris CD, Carlson SA, Dorn JM, Fulton JE. Disparities in adolescents' residence in neighborhoods supportive of physical activity-United States, 2011-2012. MMWR Morb Mortal Wkly Rep. 2016;65(23):598-601. PubMed ID: 27309671 doi:10.15585/mmwr. mm6523a2

23. Li S, Treuth MS, Wang Y. How active are American adolescents and have they become less active? Obes Rev. 2010;11(12):847-862. PubMed ID: 19874527 doi:10.1111/j.1467-789X.2009.00685.x

24. Leek D, Carlson JA, Cain KL, et al. Physical activity during youth sports practices. Arch Pediatr Adolesc Med. 2011;165(4):294-299. PubMed ID: 21135319 doi:10.1001/archpediatrics.2010.252

25. Bassett DR, Fitzhugh EC, Heath GW, et al. Estimated energy expenditures for school-based policies and active living. Am J Prev Med. 2013;44(2):108-113. PubMed ID: 23332325 doi:10. 1016/j.amepre.2012.10.017

26. Bates LC, Zieff G, Stanford K, et al. COVID-19 impact on behaviors across the 24-hour day in children and adolescents: physical activity, sedentary behavior, and sleep. Children. 2020;7(9):138. doi:10.3390/ children7090138

27. Dunton GF, Do B, Wang SD. Early effects of the COVID-19 pandemic on physical activity and sedentary behavior in children living in the US. BMC Public Health. 2020;20(1351):1-13. doi:10. 1186/s12889-020-09429-3

28. Pavlovic A, DeFina LF, Natale BL, et al. Keeping children healthy during and after COVID-19 pandemic: meeting youth physical activity needs. BMC Public Health. 2021;21(485):1-8. doi:10. 1186/s12889-021-10545-x

29. Tulchin-Francis K, Stevens W Jr, Gu X, et al. The impact of the coronavirus disease 2019 pandemic on physical activity in US children. J Sport Health Sci. 2021;10(3):323-332. PubMed ID: 33657464 doi:10.1016/j.jshs.2021.02.005

30. Committee on Physical Activity and Physical Education in the School Environment. Approaches to physical activity in schools. In: Kohl HW III, Cook HD, eds. Educating the Student Body: Taking Physical Activity and Physical Education to School. Washington, DC: National Academies Press; 2013.

31. Centers for Disease Control and Prevention. Increasing Physical Education and Physical Activity: A Framework for Schools. Atlanta, GA: Centers for Disease Control and Prevention, US Dept of Health and Human Services; 2019. https://www.cdc.gov/healthyschools/ physicalactivity/pdf/2019_04_25_PE-PA-Framework_508tagged.pdf

32. Michael SL, Wright C, Mays Woods A, van der Mars H, Brusseau TA, Stodden DF. Rationale for the essential components of physical education. Res Q Exerc Sport. 2021;92(2):202-208. PubMed ID: 33465020 doi:10.1080/02701367.2020.1854427

33. Tsuda E, Wyant J, Bulger SM, et al. Status of State-Level Physical Education Accountability Policy and Systems. Chicago, IL: Active Schools; 2019. https://www.activeschoolsus.org/wp-content/uploads/ 2020/02/Ful-Report.pdf

34. Sallis JF, Prochaska JJ, Taylor WC. A review of correlates of physical activity of children and adolescents. Med Sci Sports Exerc. 
2000;32(5):963-975. PubMed ID: 10795788 doi:10.1097/00005768200005000-00014

35. Payan DD, Sloane DC, Illum J, Lewis LB. Intrapersonal and environmental barriers to physical activity among Blacks and Latinos. $J$ Nutr Educ Behav. 2019;51(4):478-485. PubMed ID: 30442570 doi:10.1016/j.jneb.2018.10.001

36. Dishman RK, McIver KL, Dowda M, Saunders RP, Pate RR. Selfefficacy, beliefs, and goals: moderation of declining physical activity during adolescence. Health Psychol. 2019;38(6):483-493. PubMed ID: 30973746 doi:10.1037/hea0000734

37. Miller JM, Pereira MA, Wolfson J, Laska MN, Nelson TF, NeumarkSztainer D. Are correlates of physical activity in adolescents similar across ethnicity/race and sex: implications for interventions. J Phys
Act Health. 2019;16(12):1163-1174. PubMed ID: 31651411 doi:10. 1123/jpah.2018-0600

38. Thompson WM, Berry D, Hu J. A church-based intervention to change attitudes about physical activity among black adolescent girls: a feasibility study. Public Health Nurs. 2013;30(3):221-230. PubMed ID: 23586766 doi:10.1111/phn.12009

39. Troped PJ, Wiecha JL, Fragala MS, et al. Reliability and validity of YRBS physical activity items among middle school students. Med Sci Sports Exerc. 2007;39(3):416-425. PubMed ID: 17473767 doi:10. 1249/mss.0b013e31802d97af

40. Prochaska JJ, Sallis JF, Long B. A physical activity screening measure for use with adolescents in primary care. Arch Pediatr Adolesc Med. 2001; 155(5):554-559. PubMed ID: 11343497 doi:10.1001/archpedi.155.5.554 